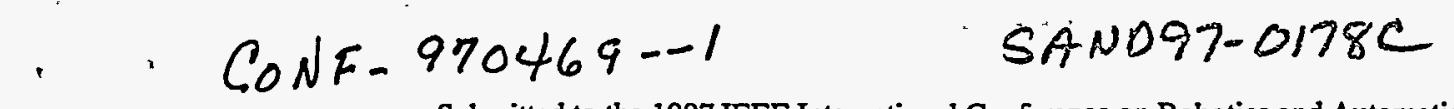

Submitted to the 1997 IEEE International Conference on Robotics and Automation

\section{A 3-d Modular Gripper Design Tool}

\author{
Russell G. Brown and Randy C. Brost* \\ Sandia National Laboratories \\ Albuquerque, NM 87185-1008
}

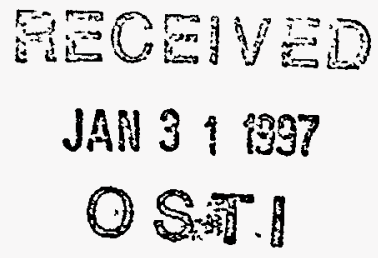

Modular fixturing kits are sets of components used forflexible, rapid construction of fixtures. A modular vise is a parallel-jaw vise, each jaw of which is a modular fixture plate with a regular grid of precisely positioned holes. To fixture a part, one places pins in some of the holes so that when the vise is closed, the part is reliably located and completely constrained. The modular vise concept can be adapted easily to the design of modular parallel-jaw grippers for robots. By attaching a gridplate to each jaw of a parallel-jaw gripper, we gain the - ability to easily construct high-quality grasps for a wide variety of parts from a standard set of hardware.

Wallack and Canny [16] developed an algorithm for planning planar grasp configurationsfor the modular vise. In this paper, we expand this work to produce a 3-d fixture/gripper design tool. We describe several analyses we have added to the planar algorithm, including a 3-d grasp quality metric based on force information, 3-d geometric loading analysis, and inter-gripper interference analysis. Finally, we describe two applications of our code. One of these is an internal application at Sandia, while the other shows a potential use of our code for designing part of an agile assembly line.

\section{Introduction}

Part holding is a fundamental problem in automated manufacturing. Fixturing is a requirement for many manufacturing processes, including machining, assembly, and inspection. For machining purposes, fixturing is necessary to immobilize the part against tool forces. For assembly, the part must be immobilized against insertion, fastening, and pallet transfer forces. For inspection tasks, the part must be located accurately. Automated grasping is also fundamental to assembly processes.

Many commercial firm' face increasing pressure to bring products to market quickly. This motivates the development of ways to speed the implementation of production processes. In this paper, we present a way to - speed the design and construction of robot grippers.

We base our work on the results of Wallack and Canny $[16,17]$, who developed an algorithm for automatically designing planar fixtures for a modular vise. This is a parallel-jaw vise, where the top of each jaw is a fixture-plate with a grid of holes. To fixture a part, one places four cylindrical pins in selected grid holes, and then closes the vise to constrain the part. By applying this concept to the jaws of a parallel-jaw gripper, we gain the ability to design high-quality grasps for a wide variety of parts from a common set of hardware.
The grasps produced by the Wallack and Canny algorithm hold the part in planar form closure. In other words, the part is constrained against external disturbance forces in the $x y$-plane or torques about the $z$-axis without friction. The output of Wallack and Canny's modular vise fixture design algorithm is the set of all two-dimensional fixtures which provide twodimensional form closure for a two-dimensional part. The fixturing elements are circles, and the part features are line segments or circular arcs in the $x y$-plane. For a review of the algorithm, see $[16,17]$ or [4].

In this paper, we present several extensions we have made to the modular vise algorithm. We have added the ability to design fixtures for three-dimensional parts. This is done by extracting legal contact features from 3-d solid CAD models and by analyzing the CAD models to determine heights for the fixture elements. In addition, we have added an analysis that determines whether a given fixture is easy to load. This determines whether it is possible to insert the part into the open vise with nonzero clearance, and then close the vise to obtain the desired grasp. We have also added the ability to analyze the behavior of a fixture under a given set of applied disturbance forces, which in turn gives us a quality metric based on how well the fixture will resist the expected disturbance forces. This necessarily requires an analysis of contact friction, as we shall see later. Finally, we have also implemented an inter-gripper interference analysis to determine the compatibility of multiple grasps for handing off the part from one gripper to another.

The following sections explain these extensions in detail. Section 2 provides a brief review of related fixturing, grasping, and mechanics analysis literature. Section 3 provides a detailed description of the algorithms and analyses that we have added to Wallack and Canny's work. Section 4 describes two applications of our algorithms to real problems. Finally, in Section 5, we summarize the lessons we've learned in the course of this research and observed limitations to this work.

\section{Related Research}

The work we present here should be considered in the context of existing research from both the fixturing and grasping fields. As with much of the work in these areas, we draw extensively on research from the force analysis and mechanics field. We can only touch on the relevant literature here, and refer the reader to [4] for a more complete treatment of previous work, and to [6] for a survey of gripper designs and grasp analysis methods.

\footnotetext{
*Supported by US DOE contract DE-AC04-94AL85000.
}
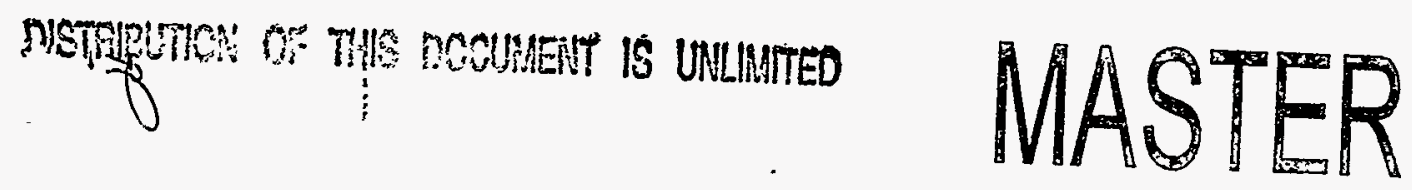
Salisbury [9] describes analysis of multifingered hands from a kinematic and grasp force perspective; our force-based quality metric is based on Salisbury's formulation. Nguyen reports several analytic results regarding force closure and a grasp synthesis algorithm in [12]; these results helped clarify the ambiguity issue addressed in Section 3.5.2. Other analytic results include Trinkle's quanitative measure of form closure [14] and De Meter's analysis of assembly fixtures [10]; these results both employ linear programs that share common features with our grasp quality measure. See [2] for a recent example of an alternative approach to assessing grasp quality.

Our algorithm designs grasps that provide planar form closure, but not 3-d form closure. For proofs addressing the number of contacts required for $3-\mathrm{d}$ form closure, see Mishra, et al [11], and Markenscoff, et al [8].

Several works in the fixturing literature provide synthesis algorithms which use some sort of modular hardware kit to promote ease of implementation of the fixtures produced by the algorithms, and also to constrain the space of possible fixtures which the algorithm must search. Recent examples include algorithms reported by Kim [7] and Brost and Peters [3], which generate 3-d fixture plans using modular locators and clamps, considering geometric and force constraints. These algorithms are similar to ours, especially in their geometric analysis.

Ponce has described a three-dimensional modular fixturing vise and an algorithm to design fixtures for it [13]. Ponce's fixturing device differs from ours in that the fixture plates are on the inner faces of the jaws, while in our vise, the fixture plates are on the top of the jaws.

\section{Design and Analysis Algorithms}

\subsection{Problem Statement}

Our primary assumption is that the workpiece and all fixture elements are rigid bodies. We require the user to provide the workpiece in the desired vertical orientation we assume that the vertical axis of the workpiece does not change. Our quality analysis assumes Coulomb friction.

The input to this tool consists of the following:

- An ACIS ${ }^{\circledR}$ CAD model of the part to be fixtured, including material specification.

- A fixture kit specification: the grid spacing, pin radius, pin material, jaw travel limits of the gripper, and maximum available squeezing force.

- A table of friction coefficients $\mu$, indexed by material pair.

- A list of the forces that will be exerted on the part while it is held in the gripper.

Given this input, the algorithm outputs a series of valid fixture designs. Each output design is geometrically feasible and obeys the jaw travel limits. Further, each fixture is easy to load - the gripper fingers can close from the open position to the grasp position without interference. Finally, all returned fixtures can resist the expected disturbance forces, without exceeding the available squeezing force. Fixtures are given quality scores according to the excess squeezing force that is available, and output in order of decreasing quality.

\subsection{Algorithm Synopsis}

1. Extract 2-d projected edges from the 3-d model.

2. Generate planar fixtures.

3. Convert planar fixtures into 3-d fixtures.

4. Analyze ease of loading.

5. Perform force-based quality analysis.

\subsection{3-d Fixture Synthesis}

Our code interfaces to $3-\mathrm{d} C A D$ data using the ACIS solid modeler. Using the ACIS data structures, we extract line segments and circular arcs from the part, keeping only those features which are accessible to the gripper.

Our method for extracting 2-d features is the following: We extract from the ACIS model all linear edges and those elliptical arcs whose projections onto the $x y$ plane are approximately circular. In addition, we extract linear edges formed by projecting cone sections onto the $x y$-plane. We project these features onto the $x y$-plane and then find all intersections between pairs of features. We break the features into sections (edgelets) at the intersections. We then determine, for each edgelet, whether it is visible from below. We determine this using the ACIS ray-shooting facility. A ray is shot straight upward from below the part at the $(x, y)$ location of the edgelet's midpoint. If that ray first intersects the part at the feature which generated the edgelet, then the edgelet is visible from below. If the ray intersects the part at a lower height, then the edgelet is occluded, so we discard it. Once we have filtered the edgelets, we recombine visible, adjacent edgelets from the same original feature. The combined edgelets are then passed to the planar fixture generation algorithm. Since our implementation of the planar algorithm only accepts linear edges, at this time we convert all circular arcs to piecewise linear approximations.

The limited set of geometric feature types used in the initial projection affects the completeness of the algorithm, but not its correctness, as any locator pin which intersects a part feature of an unhandled type will be detected later in the algorithm. If the object has only planar faces, then the algorithm is complete and generates all feasible fixture designs. If the object contains other surface types such as NURBS patches, then some valid fixtures may be overlooked, but no incorrect fixtures will be returned.

After we generate the planar fixtures, we convert each planar fixture to a 3-d fixture by computing appropriate heights for the pins. First we transform the part into its fixtured pose. Next, for each pin, we use the ACIS ray-shooting facility to determine the contact heights pin-contact-min and pin-contact-max, and in the case of non-silhouette edges, pin-height-max. For a pin of diameter $d$, we shoot rays of diameter $d+\epsilon$ or $d-\epsilon$ to assure or prevent intersection 


\section{DISCLAIMER}

This report was prepared as an account of work sponsored by an agency of the United States Government. Neither the United States Government nor any agency thereof, nor any of their employees, makes any warranty, express or implied, or assumes any legal liability or responsibility for the accuracy, completeness, or usefulness of any information, apparatus, product, or process disclosed, or represents that its use would not infringe privately owned rights. Reference herein to any specific commercial product, process, or service by trade name, trademark, manufacturer, or otherwise does not necessarily constitute or imply its endorsement, recommendation, or favoring by the United States Government or any agency thereof. The views and opinions of authors expressed herein do not necessarily state or reflect those of the United States Government or any agency thereof. 


\section{DISCLAIMER}

Portions of this document may be illegible in electronic image products. Images are produced from the best available original document. 

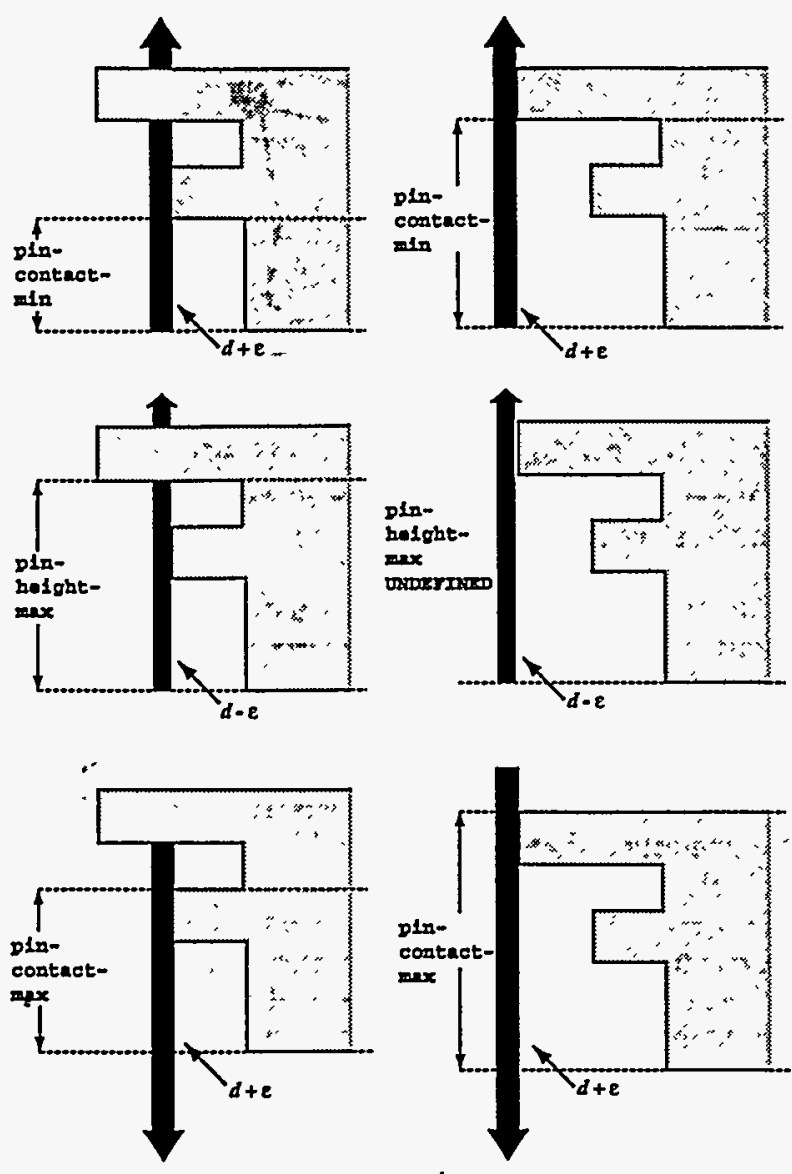

Figure 1: Determining pin heights. On the left, the case where the pin touches a nonsilhouette edge of the part, visible only from below. On the right, the silhouette edge case.

with desired contact surfaces, as shown in Figure 1. The final pin height is chosen slightly shorter than pin-height-max for non-silhouette edges, and slightly taller than pin-contact-max for silhouette edges. If pin-contact-min and pin-contact-max are essentially equivalent, then we provide the grasp quality analysis with a single contact point for that pin. If the two heights are distinct, then we provide the analysis with two discrete point contacts, which are functionally equivalent to any combination of point and line contacts on the line segment connecting the two extremal contact points.

\section{- 3.4 Loading Analysis}

The basic 2-d modular vise algorithm returns all grasps that provide planar form-closure. Unfortunately, some grasps returned by the algorithm cannot be easily achieved, because they require a complex rotating motion to acquire the part, or a tight-tolerance insertion to reach the grasp configuration. We prefer grasps which may be attained by simply opening the gripper to full extent, placing it around the part, and then closing the gripper until the applied force is resisted by the material of the part being grasped. We refer to such grasps as loadable and use a filter to select only those grasps:

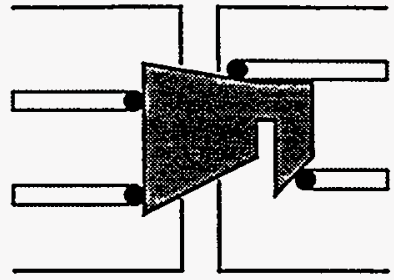

(a)

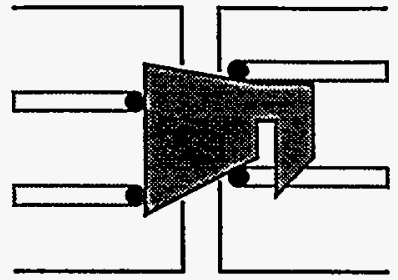

(b)
Figure 2: The loadability test: (a) loadable. (b) not.

We verify that edge normals point to the left for edges touching left-jaw pins and to the right for edges touching right-jaw pins. For each such grasp, we verify that the pins can move from their fully-opened position to the grasp position without intersecting the part. We do this by determining whether a set of axis-aligned rectangular prisms intersects the part, as shown in Figure 2.

\subsection{Force Analysis}

We have implemented a grasp quality metric that considers the three-dimensional shape of the grasped part. All grasps returned by the 2-d fixture generation algorithm provide planar form-closure - no reliance is placed on friction for holding the part in the plane. However, for 3-d gripper design we cannot use form-closure as an indication of grasp adequacy, for two main reasons.

One reason is that we clearly cannot provide 3-d form closure with parallel, vertical, frictionless cylinders. We could obtain full six degree-of-freedom form closure by adding supports and top clamps (Figure 3a), or by adding hooks to the ends of the pins (Figure $3 \mathrm{~b}$ ), but if the gripper has to pick parts off of a level surface or insert parts into holes, these approaches appear infeasible. We must use friction to hold the part.

The second reason for looking beyond form closure to determine grasp quality is this: form closure assumes rigidity and the ability to apply forces of arbitrary magnitude. For robotic grippers, however, these are not realistic assumptions.

\subsubsection{A Force-Based Quality Metric}

We want to maximize the margin for error provided by a gripper at its rated maximum squeezing force. This is equivalent to minimizing the squeezing force required to hold the part against all disturbance forces. We therefore measure grasp quality from the set of disturbance wrenches which are likely to be encountered by the grasp in its intended application. These can be determined by examining the operations that take place in the application. For example, if a pin is to be inserted in a given position on the held part, then the insertion forces caused by the pin, applied at the insertion point, are appropriate disturbance wrenches for that operation. For each candidate grasp, we compute the minimum squeezing force $F_{s q}$ required to resist each disturbance wrench. The maximum $F_{s q}$ over all of the disturbance wrenches becomes the quality measure for the grasp. The best grasps, then, are the ones which require the smallest squeezing force to be able to resist all disturbance wrenches. 


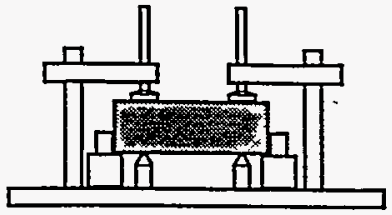

(a)

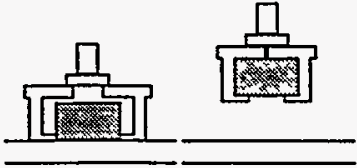

(b)
Figure 3: (a) One means of obtaining 3-d form-closure with modular elements: in addition to the side locators, support pads and top clamps are used to constrain the part. (b) This approach isn't feasible for parallel-jaw grippers - how do we go from the picture on the left to the one on the right?

We have used a rigid-body mechanics approach to determining the minimum gripper force required to oppose the expected disturbance wrenches. The metric accepts a candidate grasp, a set of expected disturbance wrenches $\mathcal{W}$, and a force limit $F_{l i m i t}$ describing the maximum squeezing force that may be exerted by the gripper. Given this input, the metric calculates the squeezing force $F_{m a x}$ required to counter the worst-case wrench in $\mathcal{W}$. If this value is greater than the maximum available squeezing force $F_{\text {limit }}$, then the grasp is infeasible and we discard it. Otherwise, $F_{\max }$ becomes the basis for comparing alternative fixture designs; designs with the smallest $F_{m a x}$ are preferred because these provide the greatest margin of safety relative to the maximum available squeezing force $F_{\text {limit }}$.

\subsubsection{Squeeze Force Calculation}

The key calculation required to compute this metric is the calculation of the squeezing force $F_{s q}$ required to resist a given 6-dof disturbance force $F_{D}$, expressed in the form $\left[\begin{array}{llllll}f_{x} & f_{y} & f_{z} & \tau_{x} & \tau_{y} & \tau_{z}\end{array}\right]^{T}$. This calculation is done for each $\mathbf{F}_{D} \in \mathcal{W}$. The maximum $F_{s q}$ is taken to be $F_{\text {max }}$. We now consider how $F_{s q}$ may be calculated. This calculation must include friction, since motion in the $\pm z$ direction cannot be resisted without friction.

We would like to establish the minimum $F_{s q}$ that would guarantee that a given grasp will resist a given disturbance $\mathbf{F}_{D}$. Unfortunately, because of the ambiguities that are present in Newtonian rigid-body dynamics with Coulomb friction, this problem is known to be NP-complete in 2-d and remains open in 3-d $[1,5,15]$. Consequently, we instead compute the minimum gripper force $F_{s q}$ which can oppose the disturbance $\mathrm{F}_{D}$ in static equilibrium. This leaves open the possiblity of object motion in ambiguous cases.

We partially address this concern by disallowing situations where contacts with the fingers on a single jaw can produce force closure with no participation by contacts on the second jaw. Such grasps suffer from wedging, which has a number of disadvantages. For example, the grasp shown in Figure 4 may resist arbitrary disturbances using the contacts on the left jaw alone, depending on the internal strain created by these contacts. (Consider the effect of wedging the part tightly between the fingers, like a door stop.) Since our rigid-body model does not represent this internal strain, this case may lead

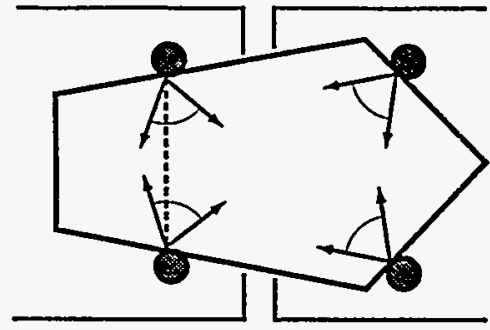

Figure 4: A grasp which provides frictional force closure with a single jaw. Because the dashed line connecting the contacts on the left lies within the friction cones at each contact, these contacts alone provide force closure [12].

to ambiguities. We discard such fixtures, thereby decreasing the likelihood of encountering an ambiguity.

\section{Modeling Contact and Squeezing Force}

For each of the four pins on the gripper, we effectively have either one or two point contacts with the part. If the pin contacts a single edge at a point, then there is only a single point contact. If the pin contacts two or more edges or contacts one or more surfaces along a line, then we can treat this as two point contacts - the highest and lowest contacts on the pin. Thus we have between four and eight point contacts with friction, which we treat as hard finger contacts: the finger can impart no torque about the contact normal [9].

Figure 5 shows a modular vise holding a part. In this case, all pins have a single line contact with the part, giving us eight point contacts to consider. The figure also shows the contact points and the approximate directions of the contact reaction forces induced by these contacts, along with a disturbance force, $\mathrm{F}_{D}$. If the part is in equilibrium under these nine force vectors, then it must be the case that the vector sum of the nine forces, $\sum_{i} F_{i}+F_{D}$, is zero.

The total squeezing force exerted in such an equilibrium is the sum of the forces exerted by the fingers parallel to the actuation axis of the vise, and in the actuated direction for each finger. In this case, this means that, for the left two pins, the contribution to the total squeezing force is the $x$-component of $\mathrm{F}_{1}$ through $\mathrm{F}_{4}$. For the right two pins, the contribution is the negative of the $x$-component of $\mathbf{F}_{5}$ through $\mathrm{F}_{8}$. In general, if the gripper closes parallel to the $x$-axis, then the contribution of $\mathrm{F}_{i}$ toward the squeezing force $F_{s q}$, is the $x$-component of $F_{i}$ times $\sigma(i)$, where we define $\sigma(i)$ to be 1 for contacts on the left jaw and -1 for contacts on the right jaw, so that $F_{s q}=\sum_{i} \sigma(i)\left[\begin{array}{lll}1 & 0 & 0\end{array}\right] \cdot F_{i}$.

To determine whether the static equilibrium obeys friction constraints, we must break the contact reaction forces into their normal and tangential components and apply the rules of Coulomb friction. For each contact $i$, $F_{i}$ is the total force at that contact point. Let $F_{T, i}$ be the tangential force due to friction at the contact point, while $\mathbf{F}_{N, i}$ is the normal component of the total force. Coulomb's Law requires $\left|\mathrm{F}_{T, i}\right| \leq \mu\left|\mathrm{F}_{N, i}\right|$, where $\dot{\mu}$ is the coefficient of friction. 


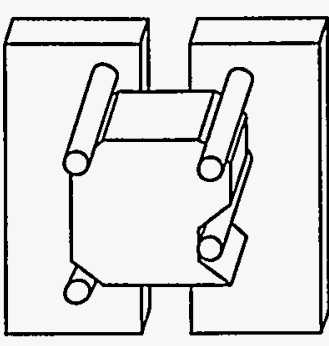

Squeezing Direction

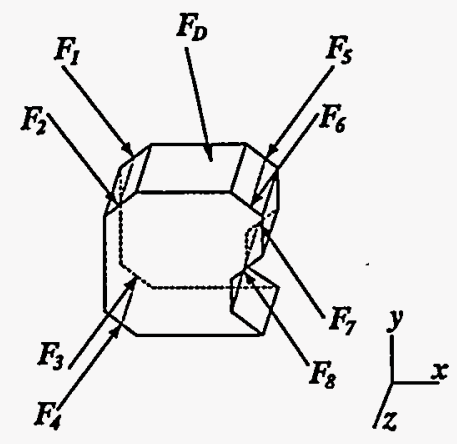

Figure 5: At left, a three-dimensional object held in a paralleljaw gripper with cylindrical pins. At right, the forces acting upon the object. $F_{1}$ through $F_{8}$ are the equivalent point contact forces caused by the line contacts between the object and the pins. $F_{D}$ is an external disturbance force acting on the object.

We also define $\mathbf{F}_{T_{x y, i}}$ and $\mathbf{F}_{T_{z}, i}$. These are the components of each $\mathbf{F}_{T, i}$ which are parallel to the $x y$ plane and orthogonal to it, respectively. We now describe a formulation of force closure which provides a way to calculate the minimum squeezing force consistent with static equilibrium under Coulomb friction.

\section{Linear Program Formulation}

In Chapter 5 of [9], Salisbury describes a method that uses the wrench representation to determine whether a given grasp resists a given disturbance wrench. For a set of $n$ frictional hard-finger contact points on an part, we apply this method starting with a set of $3 n$ unit wrenches which decompose each contact force into its normal and tangential components. These $3 n$ wrench vectors are arranged as a 6 by $3 n$ matrix $W$, with the normal-force wrenches $\mathbf{F}_{N, i}$ forming the first $n$ columns, the horizontal tangential wrenches $\mathrm{F}_{T_{x y}, i}$ in the second $n$ columns, and the vertical tangential wrenches, $\mathbf{F}_{T_{x}, i}$ in the third $n$ columns. A grasp can resist a disturbance wrench $\mathbf{F}_{D}$ if there exists a $3 n$-element vector of contact wrench magnitudes $\mathbf{c}$, such that:

- $\mathbf{W c}+\mathbf{F}_{D}=\mathbf{0}$,

- The first $n$ elements of $c$ are positive (a contact can push but not pull), and

- The magnitudes of the tangential-force wrenches at each contact do not exceed $\mu$ times the magnitude of the normal-force wrench.

We can then formulate the minimum squeezing force problem as an optimization problem, where we want to minimize $F_{s q}$ given the above conditions. Unfortunately, the third condition above is nonlinear, since it requires computing the magnitude of $F_{T, i}$. Since we don't have a general method for solving nonlinear optimization problems, we linearize the problem using the method proposed by Trinkle, et al [15]: Instead of restricting the magnitude of the total tangential force to no more than $\mu$ times the normal force, we restrict each of $\mathrm{F}_{T_{x y}, i}$ and $\mathbf{F}_{T_{x}, i}$ to be no more than $\frac{\mu}{\sqrt{2}}$ times the normal force. Thus, instead of restricting the contact force to lie in a circular cone, we restrict the force to lie in a square pyramid inscribed inside the circular cone.

This approximation allows us to write the following linear program:

minimize

$$
F_{s q}=\sum_{i} \sigma(i)\left(\left[\begin{array}{lll}
1 & 0 & 0
\end{array}\right] \cdot\left(\mathrm{F}_{N, i}+\mathrm{F}_{T, i}\right)\right)
$$

subject to

$$
\begin{aligned}
& \text { 1: } \mathbf{W c}+\mathrm{F}_{D}=0 \\
& \text { 2: } \forall i, c_{i} \geq 0 \\
& \text { 3: } \forall i,\left|c_{2 i}\right| \leq \mu^{\prime} c_{i} \\
& \text { 4: } \forall i,\left|c_{3 i}\right| \leq \mu^{\prime} c_{i}
\end{aligned}
$$

where $\mu^{\prime}=\frac{\mu}{\sqrt{2}}$, and $i \in[1, n]$, where $n$ is the number of contact points. Condition 1 ensures that static equilibrium is possible. Condition 2 ensures that each contact normal force is non-negative, and conditions 3-4 ensure that the total contact force lies within the friction pyramid. Solving this linear program identifies the minimum squeezing force for which the system can maintain static equilibrium while obeying our approximated friction constraints.

We repeat this for all expected disturbance forces and take the maximum to obtain the desired worst-case required squeezing force $F_{\max }$.

\section{Applications}

\subsection{Automated Component Cleaning Workcell}

The Automated Component Cleaning project is an initiative within Sandia's Intelligent Systems and Robotics Center to design a workcell capable of performing flexible, high-quality cleaning of machined parts using an environmentally benign alcohol spray. The workcell uses a robot arm equipped with a focused spray-nozzle to clean the parts, using an automatically formulated spraying motion to clean machine oil and similar impurities out of holes and concavities on the part.

Because an alcohol cleaning spray is used, the pallet-borne part enters the workcell through an airlock. Once the part is in the airlock, a two degree of freedom arm (one linear, one rotational) extracts the part from its pallet, carries it into the workcell, and holds it during the initial cleaning. After the first cleaning cycle, there is a handoff to a second, pedestal-mounted gripper, so that features previously inaccessible to the sprayer can be cleaned. The arm then retrieves the part from the pedestal, and returns it to the pallet in the airlock. The workcell is designed to clean a wide variety of parts, with cleaning motions designed automatically. For this reason, it was decided that the part-handling should also be fiexible and automatically designed. A system of modular grippers and locators was chosen for the arm and pedestal grippers and the pallet. 


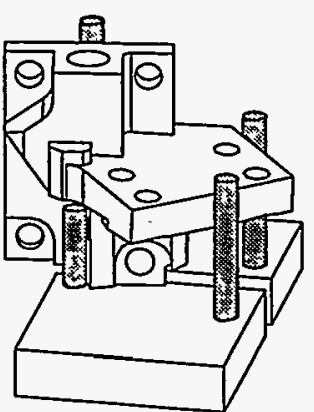

(a)
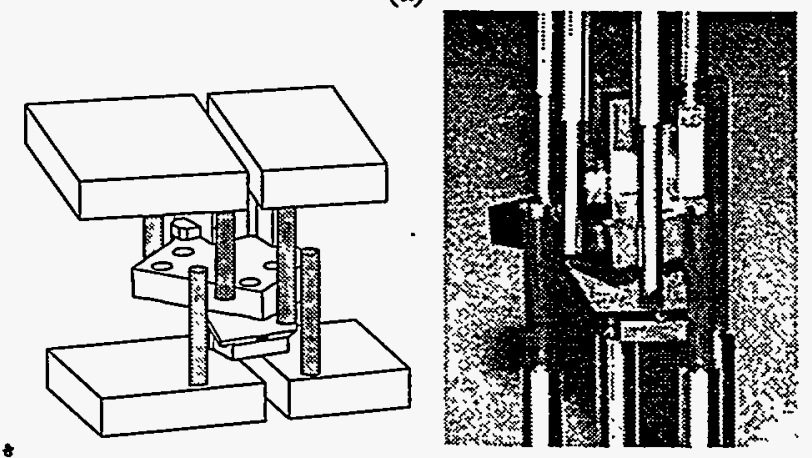

(b)

Figure 6: (a) The pedestal gripper holding the part. (b) The handoff between the arm and pedestal grippers. The pin lengths were extended to allow spray clearance from low angles.

Our automatic design program begins by generating candidate grasps for the arm gripper, pedestal gripper, and pallet. Because the part rests on a flat surface on the pallet, the pallet only needs to locate the part, not grip it. Since the part "up" orientation is the same for the pallet and the pedestal gilpper, the algorithm produces pallet designs by simply taking all pedestal gripper designs that have three fingers on one jaw, and ignoring the single finger on the other jaw.

The program then generates triples of grasps which will successfully handle the part. We use a pair-wise interference analysis to ensure that the grasps for the arm and the pallet are compatible and also that the grasps for the arm and the pedestal are compatible. This produces a series of compatible $\langle a r m$, pallet $\rangle$ and $\langle a r m$, pedestal $\rangle$ grasp pairs. A "join" operation is used to generate all triples where the arm is compatible with both the pallet and the pedestal. Figures 6 and 7 show an example grasp triple found by our code. A study of Figure 7 confirms that there is no interference between the gripper fingers during handoffs. Physical tests with the hardware verified that these grippers successfully constrain the part, and also that the handoff operation works properly.

To assist the user in selecting the best triple, our program ranks the triples by minimizing the maximum squeeze force required by the arm or pedestal grasps to resist the expected disturbances. Two types of disturbance forces act on a part in the ACC workcell. (a)

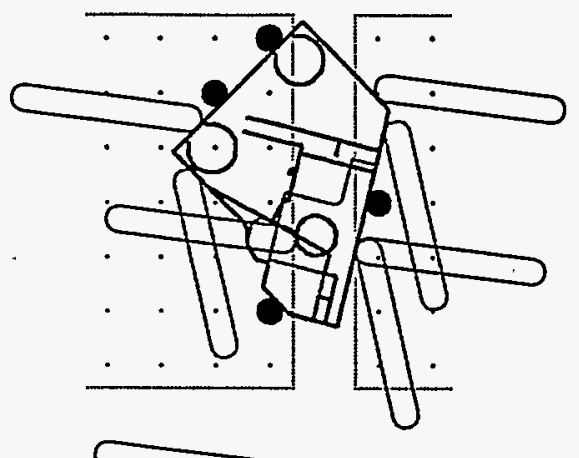

(b)

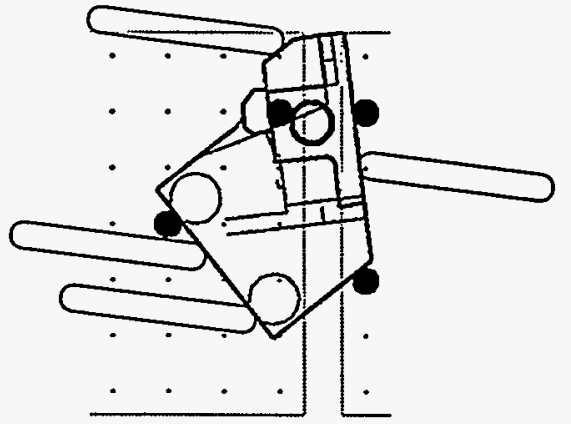

(c)

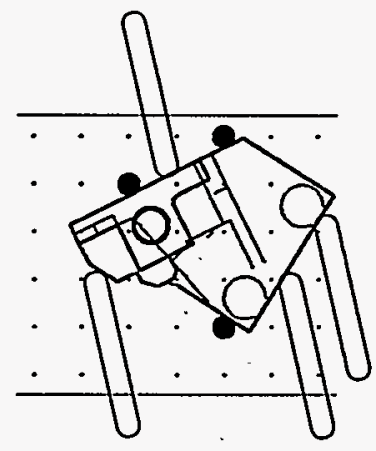

Figure 7: Top views of the ACC grippers. The "racetrack" shapes show the paths followed by fingers on the other grippers during handoff operations, showing that there is no interference. (a) The arm gripper, with the pallet (dashed) and pedestal finger paths. (b) The pedestal gripper, with the arm finger paths. (c) The pallet, also with the arm finger paths.

The first is caused by the cleaning spray. From the specifications and operating pressure for the sprayer, we calculate that the force of the spray exiting the nozzle is $4.2 \mathrm{~N}$. A conservative model of the force experienced by the part is to neglect drag and assume that the part reverses this flow, doubling the force. Thus, we use a value of $8.4 \mathrm{~N}$ for the force exerted on the part by the spray. We generate a list of expected disturbances by randomly generating a large number of points on the object surface and constructing a normal force of $8.4 \mathrm{~N}$ at each point.

The second type of disturbance force is caused by accelerations applied to the part by the arm gripper. The disturbance caused by acceleration and deceleration of the rotating arm is calculated from the mass of the part and the accelerations of the arm. The largest of these accelerations occur when the arm's joints reach their 


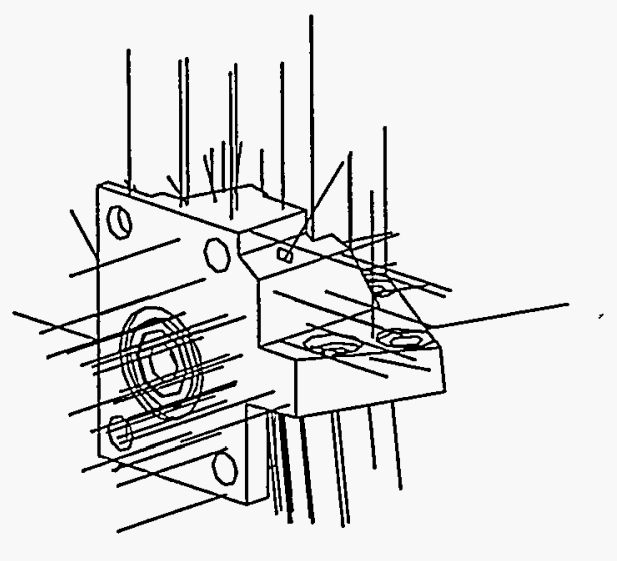

Figure 8: Disturbance forces used for grasp quality analysis.

stops. The mass of the example part is $0.18 \mathrm{~kg}$, and the maximum accelerations are strictly less than 6 gravities $\left(59 \frac{\mathrm{m}}{\mathrm{sec}^{2}}\right)$, giving maximum disturbance forces of $11 \mathrm{~N}$ acting on the centroid of the part in the $\pm z$ direction. Figure 8 shows the set of forces used for the gripper squeeze force analysis. Given a measured friction coefficient of 0.2 , the worst-case squeezing force required to resist these disturbances is $77 \mathrm{~N}$ for the arm grasp, and $39 \mathrm{~N}$ for the pedestal grasp, which does not incur the $11 \mathrm{~N}$ acceleration.

\subsection{Fixture Loading and Unloading}

In this section, we describe the use of modular grippers for flexible loading and unloading of assembly fixtures. Consider the beginning and the end of an automated assembly line: At the beginning of the line, a stream of some base part (e.g, a chassis or housing) is presented to the line for assembly, perhaps on a series of trays. The base parts need to be removed from the trays and placed into assembly fixtures, so. that they can be fed through the line, with some assembly operation occurring at each station. Similarly, at the end of the line, the assembled or semi-assembled product must be transferred from the fixtures into trays or boxes. The gripper used to load and unload these fixtures must avoid interference with the fixture. At the end of the assembly process, additional constraints on the grasp result from the presence of attached parts which may not be rigid enough to permit grasping of the assembly by those parts.

We have implemented code which takes a description of a fixture, along with descriptions of the base part and of any attached parts which create constraints, and produces a list of gripper designs which can be used to load or unload the fixture. By treating the attached parts as constraint regions, we can design a gripper that can load the part into the fixture at the start of the assembly, and also remove it from the fixture at the end. Further, the design tool can search for common gripper designs which can load and unload a variety of different parts, with no hardware change. This can be useful for flexible assembly lines which have frequent product changeovers, perhaps among several related products.
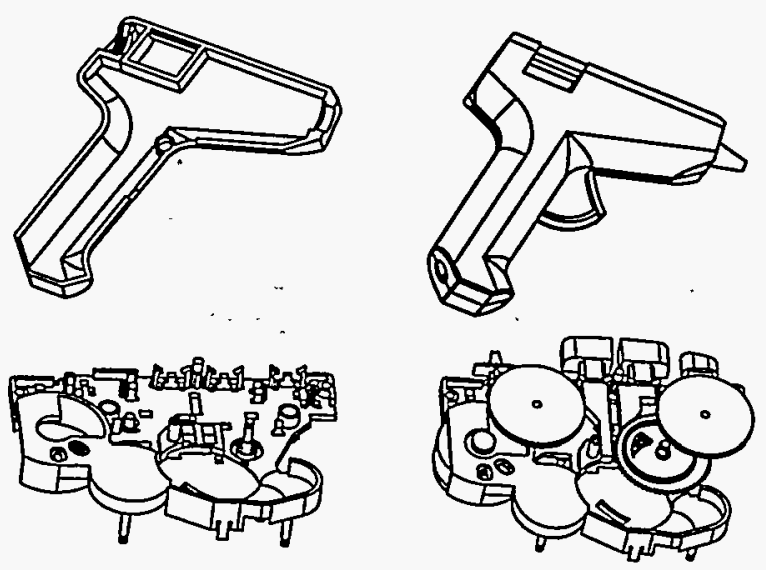

Figure 9: The base parts and assemblies used in the fixture loading/unloading example.

We now describe an example of this application in which a single modular gripper is automatically designed which can load or unload either of two products from their assembly fixture. The example products are a personal cassette chassis and a plastic hot-melt glue gun.

Figure 9 shows one shell of the glue gun and the cassette chassis before and after assembly. We ran our code on the glue gun with a $3 / 8$ " grid spacing and $1 / 4$ " diameter pins and obtained 24,367 grasps which could successfully pick up the empty housing. 5,563 of these could also successfully remove the finished assembly from the fixture. For the empty cassette chassis, the code produced 6,753 grasps. Because much of the chassis perimeter is occupied by gears, buttons, and the side locators of the fixture, only 48 of these grasps could remove the chassis from the fixture after assembly. The common grasp code identified three 〈gluegun-grasp, cassette-grasp〉 pairs, one of which is shown in Figures 10 and 11. Physical tests verified the success of this design over repeated trials (Figure 11).

\section{Conclusion}

The algorithms described in this paper extend Wallack and Canny's planar modular vise algorithm. We have provided an interface to 3-d CAD models and code to convert planar fixtures into 3-d grippers with proper length fingers. Loadability analysis ensures that the returned grasps can be easily achieved. We have added a force analysis that takes a gripper design and a set of expected disturbance forces and returns the minimum squeeze force that can oppose all of the expected disturbances. To quickly find the best grasps, we rank them using this minimum squeeze force.

We have applied our code to two examples. In the first example, we used the code to design triples of non-interfering grasps for the Sandia Automated Component Cleaning workcell. In the second example, we used the code to design a gripper for loading and unloading assembly fixtures on a mixed-product assembly line. We performed physical tests to verify the success of the resulting designs in both cases. 

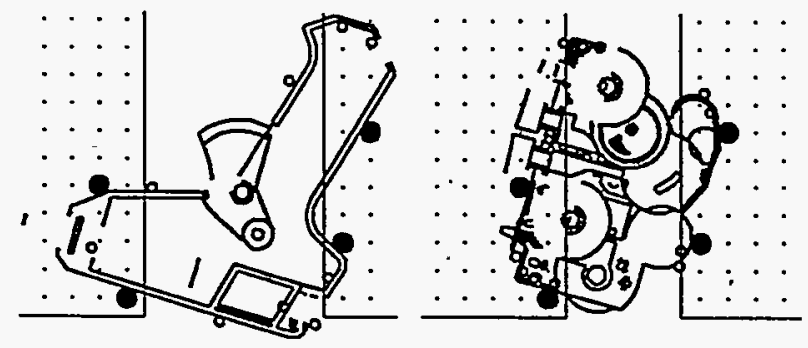

Figure 10: The common gripper for the gluegun and the cassette chassis. The views shown are in the gripper frame of reference, as though seen through transparent plates. The small open circles are the pins of the assembly fixture.
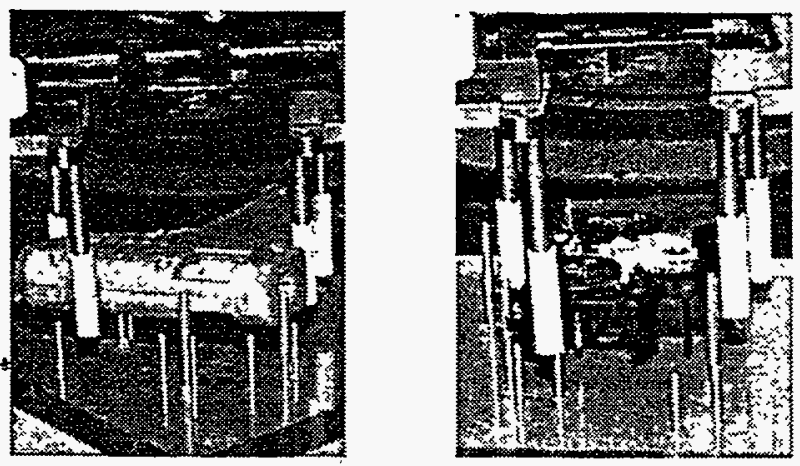

Figure 11: The glue gun and the cassette chassis held by the common gripper shown in Figure 10.

In the course of this research, we've spent substantial time considering the possibilities offered by modular parallel-jaw grippers, and also talking with automation engineers from industry. The biggest reaction we've had from our industrial contacts is concern about the cylindrical gripper fingers. The problem is primarily one of synthesizing grippers that do not interfere with the surrounding assembly during insertion operations. In many cases, it is impossible to select a diameter of cylindrical finger which will provide sufficient rigidity while avoiding interference during the insertion. Possible solutions to this problem might involve the use of an expanded set of finger primitives, such as families of flat, circular arc, or angled corner pieces.

Another limitation on both the analysis we have performed and the possibilities offered by the algorithm is that we assume that a part being grasped contacts only the fingers of the gripper, and only has contact with vertical surfaces on the fingers. While we have already observed that it is not feasible to use horizontal contacts to completely constrain the part, it is reasonable to use them to add stability. For example, we could equip the fingers with shoulders that can oppose disturbance forces with a significant component directed toward the palm of the gripper. Another possibility would be to account for the stabilizing effect of placing the part against the palm of the gripper.

\section{Acknowledgments}

We thank Aaron Wallack for providing his planar fixture design code, and Ralph Peters and Randy Wilson for comments on a draft of this paper.

\section{References}

[1] D. Baraff. Determining frictional inconsistency for rigid bodies is np-complete. Technical Report TR 90-1112, Cornell University Department of Computer Science, April 1990.

[2] A. Bicchi. On the closure properties of robotic grasping. Intl. Journal of Robotics Research, 14(4):319-334, August 1995.

[3] R. C. Brost and R. R. Peters. Automatic design of 3-d fixtures and assembly pallets. In IEEE Intl. Conference on Robotics and Automation, pages 495-502, April 1996.

[4] R. G. Brown and R. C. Brost. A 3-d modular gripper design tool. Technical Report SAND 96-????, Sandia National Laboratories, November 1996.

[5] M. Erdmann. On a representation of friction in configuration space. Inth. Journalof Robotics Research, 13(3):240 271, June 1994.

[6] R. A. Grupen, T. C. Henderson, and I. D. McCammon. A survey of general purpose manipulation. Intl. Journal of Robotics Research, 8(1):38-62, February 1989.

[7] K. H. Kim. A System for Automated Fixture Planning with Modular Fixtures. PhD thesis, Carnegie Mellon University Robotics Institute, May 1993.

[8] X. Markenscoff, L. Ni, and C. H. Papadimitriou. The geometry of grasping. Intl. Joumal of Robotics Research, 9(1):61-74, February 1990.

[9] Matthew T. Mason and J. Kenneth Salisbury. Robot Hands and the Mechanics of Manipulation. MIT Press, Cambridge, Massachusetts, 1985.

[10] E. C. De Meter. Restraint analysis of assembly work carriers. Robotics and Computer Integrated Manufacturing, 10(4):257-265, 1993.

[11] B. Mishra, J. T. Schwartz, and M. Sharir. On the existence and synthesis of multifinger positive grips. Algorithmica, 2(4):641-558, 1987.

[12] V. Nguyen. Constructing force-closure grasps. Intl. Journal of Robotics Research,7(3):3-16, June 1988.

[13] J. Ponce. On planning immobilizing fixtures for three dimensional parts. In IEEE IntL. Conference on Robotics and Automation, pages 509-514, April 1996.

[14] J. C. Trinkle. A quantitative test for form closure grasps. In IEEE/RSJ IntL. Conference on Intelligent Robots and Systems, pages 1670-1677, July 1992.

[15] J. C. Trinkle, J-S. Pang, S. Sudarsky, and G. Lo. On dynamic multi-rigid-body contact problems with coulomb friction. Technical Report 95-003, Texas A+M University Department of Computer Science, January 1995.

[16] A. S. Wallack and J. F. Canny. Planning for modular and hybrid fixtures. In IEEE Intl. Conference on Robotics and Automation, pages 520-527, May 1994.

[17] A. S. Wallack and J. F. Canny. Modular fixture design for generalized polyhedra. In IEEE Intl. Conference on Robotics and Automation, pages 830-837, April 1996. 